\title{
Catalytic Fe-oxo clusters stabilized on the MOF-808 metal organic- framework for the degradation of water pollutants
}

Accepted 00th January 20xx

Celia Castillo-Blas, ${ }^{a}$ Ignacio Romero-Muñiz, ${ }^{a}$ Andreas Mavrandonakis, ${ }^{b}$ Laura Simonelli, ${ }^{\mathrm{c}}$ and Ana E. Platero-Prats ${ }^{\text {a, }}$ *

DOI: $10.1039 / \times 0 \times x 00000 x$

\begin{abstract}
Stabilizing catalytic iron-oxo-clusters within nanoporous metalorganic frameworks (MOF) is a powerful strategy to prepare new active materials for the degradation of toxic chemicals, such as bisphenol A. Herein, we combine pair distribution function analysis of total X-ray scattering data and X-ray absorption spectroscopy, with computational modelling to understand the local structural nature of added redox-active iron-oxo clusters bridging neighbouring zirconia-nodes within MOF-808.
\end{abstract}

The removal of emerging pollutants of chemical complexity from water sources is a global health challenge. Water quality is often compromised by the presence of a variety of toxic compounds produced by human activities - ranging from heavy metals to a wide scope of organic compounds. ${ }^{1}$ In particular, bisphenol A (BPA) is an abundant endocrine disrupting chemical (EDC) pollutant in water, which is widely used for the manufacture of plastics and epoxy resins. Over the last years, a variety of porous solids, such as zeolites ${ }^{2}$ and mesoporous carbons, ${ }^{3}$ have been used to capture BPA. On the other hand, Fenton reactions catalyzed by iron(II) are a powerful methodology to afford the oxidative degradation of this pollutant in water into mainly carbon dioxide and water. ${ }^{4,5}$ However, acidic conditions are typically required to stabilize iron species in water under catalytic conditions. Therefore, developing porous materials with intrinsic highly acidic surfaces and redox-active iron sites able to capture BPA and subsequently convert it into non-toxic compounds, would represent an elegant strategy to degrade this water pollutant under mild conditions.

Metal-Organic Frameworks (MOFs) are porous and crystalline materials composed of organic ligands linked by metal-oxo nanoclusters, to give open architectures with large surface areas and pores of different shapes and sizes. ${ }^{6}$ The exceptional textural and chemical properties of these materials, make them excellent candidates for the selectively capture and degradation of many types of hazardous chemicals. 7,8 In particular, the family of $\mathrm{Zr}(\mathrm{IV})$-MOF materials present a high thermal and

a. Departamento de Química Inorgánica, Facultad de Ciencias, Universidad Autónoma de Madrid, Campus de Cantoblanco, 28049 Madrid, Spain.

b. Electrochemical Processes Unit, IMDEA Energy, Avenida Ramón de la Sagra 3, 28935 Móstoles, Madrid, Spain.

CLAESS beamline, ALBA Synchrotron, 08290 Cerdanyola del Vallès, Spain

d. Condensed Matter Physics Center (IFIMAC), Universidad Autónoma de Madrid, 28049, Campus de Cantoblanco, 28049 Madrid, Spain.

+ Electronic Supplementary Information (ESI) available: [details of any supplementary information available should be included here]. See DOI: $10.1039 / x 0 x \times 00000 x$ chemical stability in water, ${ }^{9}$ allowing the applicability in aqueous-based degradation of pesticides, pharmaceuticals and pollutants processes. The $\mathrm{Zr}(\mathrm{IV})$-MOFs are composed of $\mathrm{Zr}_{6} \mathrm{O}_{8}$ clusters, which can be $12-, 8-$, or 6-fold connected to carboxylate organic ligands, being UiO-type, NU-1000 and MOF808 the archetypical ones, respectively. ${ }^{10}$ MOF-808 is an interesting platform to target liquid-phase applications, including capture and degradation processes. ${ }^{11}$ Its structure is built from linking unsaturated $\mathrm{Zr}_{6} \mathrm{O}_{8}$ clusters by benzene-1,3,5tricarboxilate (BTC) ligands, to give mesopores of around $20 \AA$ size. Besides its high porosity, MOF- 808 has a remarkably highly tunable structure arising from the low saturation of the $\mathrm{Zr}_{6} \mathrm{O}_{8}$ clusters. Within the octahedral $\mathrm{Zr}_{6} \mathrm{O}_{8}$ clusters, the linking positions around the equatorial plane can serve as scaffold to insert discrete functional groups, including aminacids, ${ }^{12}$ sulfates, ${ }^{13}$ and metal complexes. ${ }^{14}$ Furthermore, the presence of available hydroxyl groups within the $\mathrm{Zr}_{6} \mathrm{O}_{8}$ clusters represents an excellent platform to further modifying the MOF-808 chemistry with transition metals.

Solvothermal incorporation in MOF (SIM) materials has been widely explored to decorate $\mathrm{Zr}_{6} \mathrm{O}_{8}$ clusters, by soaking the pristine MOF into a solution containing a target transition metal precursor. ${ }^{15}$ Thus, a variety of MOF chemical modifications with catalytic transition metal sites have been reported using this method. ${ }^{16}$ In this regard, the SIM method using a redox-active transition metal such as iron, would be a promising approach for tailoring MOF catalytic properties towards the oxidative degradation of organic EDC pollutants.

In this work, we show the chemical modification of MOF-808 with binuclear iron clusters, active for the selective capture and subsequent catalytic degradation of BPA via Fenton reactions. Detailed synchrotron characterization including pair distribution function (PDF) analysis of X-ray total scattering data and Fe $K$-edge $X$-ray absorption spectroscopy (XAS) together with density functional theory (DFT) computational modelling are applied to understand the local structural nature of the added redox-active iron clusters within MOF-808.

The SIM synthesis of Fe-MOF-808 was performed by immersion of pristine material, previously activated, in a solution containing $\mathrm{FeCl}_{2}$ in $\mathrm{N}, \mathrm{N}^{\prime}$-dimethylformamide (see ESI). To explore the kinetic aspects of the iron incorporation within MOF-808, different Fe-SIM reaction times were tested using 
two different starting Fe to $\mathrm{Zr}_{6}$ molar ratios, that is 12 and 24 hours. Powder X-ray diffraction (PXRD) data collected on the activated materials showed the presence of the MOF- 808 phase for all reaction times tested, with subtle differences in relative peak intensities. Interestingly, scanning electron microscopy and energy dispersive X-rays spectroscopy (SEM-EDS) analyses indicated that the incorporation of iron within MOF-808 strongly depends on the Fe-SIM reaction time. At short reaction times (i.e. $1 \mathrm{~h}$ ), the amount of iron incorporated in MOF-808 was significantly high (i.e. Fe to $\mathrm{Zr}_{6} \mathrm{O}_{8}$ molar ratio ca. 2 using a starting $\mathrm{Fe}$ to $\mathrm{Zr}$ molar ratio of 12). However, the material obtained showed a heterogeneous distribution of iron within the crystals, as evidenced by SEM-EDS analyses (S3, ESI). When using longer reaction times, the amount of iron incorporated decreased significantly until it reaches a plateau after $16 \mathrm{~h}$, together with the occurrence of an improved homogeneity of the iron distribution within the MOF crystals. The final Fe to $\mathrm{Zr}_{6} \mathrm{O}_{8}$ molar ratio determined was of ca. 0.5 (i.e. Fe-0.5-MOF808). Similar behaviour was observed for the synthesis of the high-content Fe-1.2-MOF-808 (S4, ESI). These results demonstrated that optimal iron MOF modification is obtained at long Fe-SIM reaction times, resulting in materials with enhanced homogeneity in their chemistries and lacking of unwanted iron oxide nanoparticles as by-products.

SEM-EDS mapping elemental analyses showed a highly homogeneous distribution of iron along the MOF crystallites for both 0.5- and 1.2-MOF-808 (Fig. 1C). Additionally, EDS spectra indicated the presence of chlorine in the materials in a $\mathrm{Cl}$ to $\mathrm{Fe}$ ratio of 3.6 (Fig. S3.3-S3.4). We hypothesize that chloride could be partially replacing hydroxyl groups from the Zr-cluster. ${ }^{17} \mathrm{~N}_{2}$ isotherms revealed an expected decrease in specific surface area associated with the iron incorporation within the MOF-808 structure together with subtle variations in the pore size distribution (Fig. 1A-B and S4). Detailed analysis of the pore volume contributions showed the occurrence of a significant decrease in volume linked to the mesopores of $18 \AA$ ( $c a .50 \%)$ compared to the minor loss seen for the micropores of $12 \AA$ Fig. $1 \mathrm{~B}$ and S4). These results would suggest that the iron sites are located pointing towards the MOF-808 hexagonal channels.

PXRD data collected on both the low- and high-content Fe-MOF808 materials showed the unique presence of the Bragg peaks linked to the MOF-808 phase together with subtle differences in relative peak intensity. Pawley refinements of the diffraction data corroborated that the average MOF architecture is preserved after Fe-SIM, together with the occurrence of a minor cell expansion and peak broadening compared to the pristine system (Fig. 1D and Table S7.1). These results demonstrated that MOF-808 is a robust platform towards the incorporation of iron sites through SIM methods.

To probe the atomic local structure of the iron sites within MOF808 , we applied PDF analysis of X-ray total scattering data (XPDF). XPDF data collected on the Fe-SIM and pristine MOF-808 materials showed two major contributions at short range, that is, $\mathrm{Zr}-\mathrm{O}(\mathrm{ca} .2 .2 \AA$ ) and $\mathrm{Zr}$... $\mathrm{Zr}$ (ca. $3.5 \AA$ A ) distances characteristic of the $\mathrm{Zr}_{6} \mathrm{O}_{8}$ clusters. ${ }^{18,19}$ With the purpose of highlighting the subtle contributions in this region that are associated to the iron sites, differential analysis of the XPDF data ${ }^{20}$ were carried out by subtracting the PDF of the pristine material to that of the FeMOF-808 systems. d-PDF analysis carried out on the Fe-MOF808 materials revealed the appearance of new correlations associated with the iron-clusters at $\sim 1.9-2.0 \AA, \sim 2.3 \AA$ and $~ 3.0$ $\AA$ (Fig. 2.A). The Fe-O distance values are sensitive to the oxidation state of iron, ${ }^{21}$ being shorter for $\mathrm{Fe}(I I I)$ ( 1.85 $\AA$ ) compared to $\mathrm{Fe}(\mathrm{II})$ ( $2.05 \AA$ A). The broad d-PDF signal observed at $~ 1.9-2.0 \AA$ indicated the presence of mixed oxidation states for iron within the Fe-MOF-808 systems. In addition, the presence of chloride groups bonded to the iron sites is confirmed by the peak centred at $\sim 2.3 \AA$, associated with $\mathrm{Fe}-\mathrm{Cl}$ bonds. Interestingly, the correlation centred at $\sim 3.0 \AA$ is characteristic of $\mathrm{Fe}-\mathrm{O}-\mathrm{Fe}$ distances within edge-sharing geometries. ${ }^{22}$ Additionally, two d-PDF peaks centred at $\sim 3.3$ and $\sim 3.7 \AA$ are observed, which could be attributed to $\mathrm{Zr}$...Fe correlations. These results indicated the presence of iron-oxo clusters binding the $\mathrm{Zr}_{6}$-nodes within the Fe-MOF-808 materials, where some of the oxygen positions have been replaced by chloride, as suggested by SEM-EDS.
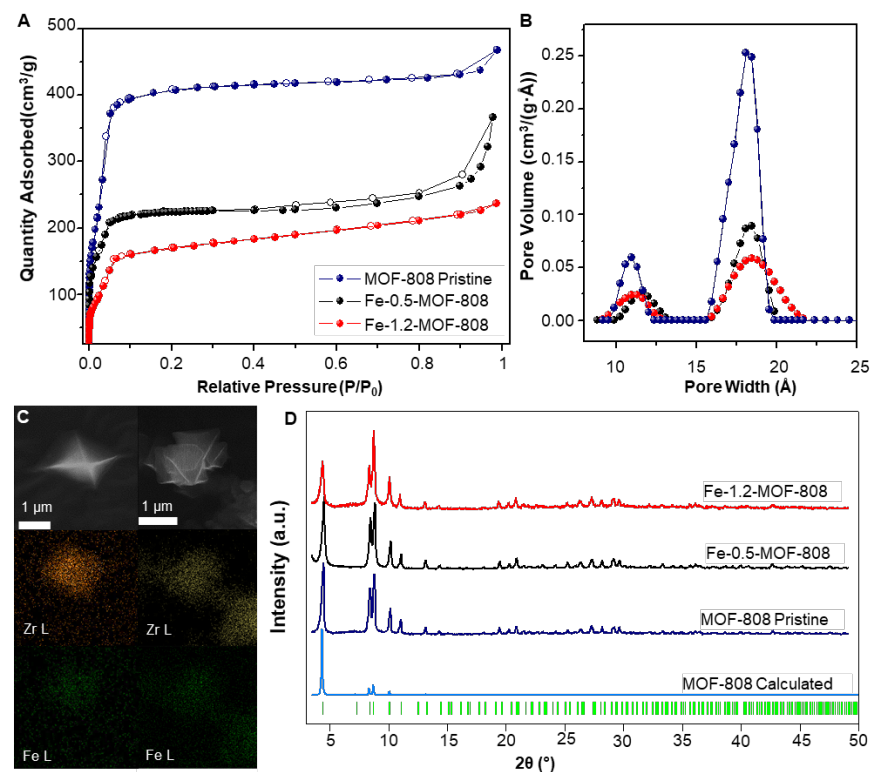

Fig. $1 \mathrm{~A} . \mathrm{N}_{2}$ adsorption/desorption isotherms at $77 \mathrm{~K}$ showing the decrease in surface area upon iron incorporation; $\mathbf{B}$. Pore size distribution (PSD) data for pristine and Fe-SIM MOF-808 materials; C. Mapping of Zr (yellow) and Fe (green) for Fe-0.5-MOF-808 (right) and Fe-1.2-MOF-808 (left); D. PXRD data of the synthesized materials and their comparison with the calculated data for MOF-808, ${ }^{23}$ including Bragg positions.

Quantitative analysis of the relative d-PDF peak intensities is a powerful strategy to estimate the size of metal-oxo clusters deposited in MOF-808. ${ }^{24}$ Thus, the signals linked to Fe-O, $\mathrm{Fe}-\mathrm{Cl}$ and Fe-O-Fe were fitted to Gaussian curves and compared to preliminary models of different iron cluster sizes (i.e. bearing two, three or four iron atoms, Fig.2.B). By comparing the experimental d-PDF peak intensities of the $\mathrm{Fe}-\mathrm{O}, \mathrm{Cl}$ and contributions to simulated values, we hypothesized that Fe-0.5MOF-808 contains clusters of two iron atoms in distorted tetrahedral environments. Furthermore, the results suggest that the iron sites are bonded to both chlorine and oxygen atom (including aquo, hydroxo or oxo group) in a 1 to 2 ratio (Figure S8.3, Table S8.1). For the Fe-1.2-MOF-808 system, quantitative analyses indicated the formation of slightly larger iron clusters compared to Fe-0.5-MOF-808 (Figure S8.4, Table S8.2), suggesting that the use of high concentrations of iron precursor during SIM on MOF-808 would favoured aggregation.

To better understand the chemical nature of the binuclear iron clusters within MOF-808, Fe $K$-edge extended X-ray absorption fine 
structure (EXAFS) and X-ray absorption near edge spectroscopy (XANES) data were collected on the Fe-MOF-808 materials. XANES data showed the occurrence of rising edge and pre-edge features at ca. 7122 and ca. $7114 \mathrm{eV}$, characteristic of binuclear iron complexes. ${ }^{25} \mathrm{~A}$ slight shift of rising-edge position of $c a .1$ to higher values is observed for Fe-1.2-MOF-808 compared to Fe-0.5-MOF808 , in agreement with the oxidation of $\mathrm{Fe}^{2+}$ to $\mathrm{Fe}^{3+}$ (Fig. 3A). The observed pre-edge signal corresponds to the Fe-1s to Fe-3d electronic transition, and its intensity is indicative of the local symmetry of the iron sites. The XANES data collected on both FeMOF-808 materials suggested the appearance of tetrahedral iron geometries. ${ }^{26}$ This experimental evidence is in agreement with the most energetically favoured geometry compatible with both II/II oxidation states found for iron within these materials. Further analyses of the EXAFS data collected on Fe-0.5-MOF-808 indicated the presence of three main signals at ca. $1.35, c a .1 .81$ and $2.58 \AA$ (without phase correction), linked to $\mathrm{Fe} / \mathrm{ll}-\mathrm{O}, \mathrm{Fe}-\mathrm{Cl}(2.25 \AA)$ and Fe...Fe distances, respectively (Fig. 3B). ${ }^{27}$ Interestingly, EXAFS data of Fe-1.2-MOF-808 showed a single contribution at ca. $1.56 \AA$ (without phase correction) linked to $\mathrm{Fe}$ III-Cl,O bonds together with a significant increase in intensity of the signal linked to $\mathrm{Fe}$... Fe distances. This evidence corroborates the presence of larger clusters with an oxidized state of iron in Fe-1.2-MOF-808 compared to Fe-0.5MOF-808, in agreement with the XPDF analyses.
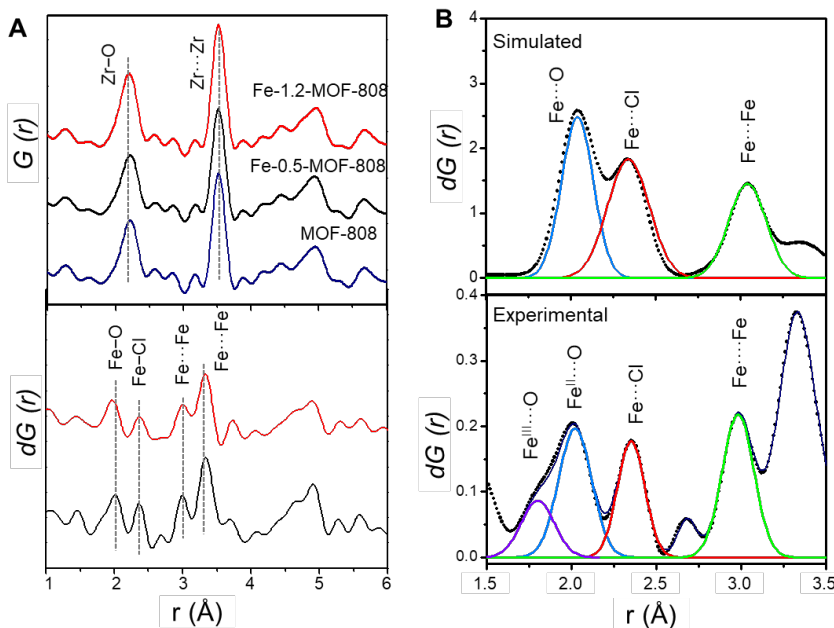

Fig. 2 A. Total PDFs of pristine and Fe-MOF-808 materials (up) and the corresponding $d$ PDFs for Fe-0.5-MOF-808 and Fe-1.2-MOF-808, obtained from the subtraction of MOF808 pristine (blue) (down); B. Simulated (up) and experimental (down) d-PDF signals linked to the iron clusters were fitted to Gaussian curves for quantitative analyses.
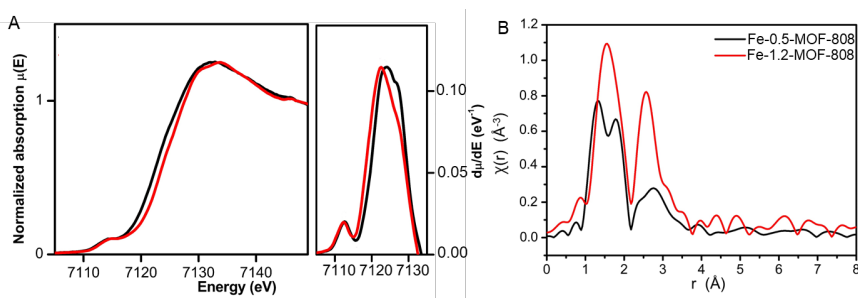

Fig. 3 A. Fe $K$-edge XANES data of Fe-MOF-808 materials (left) and first derivate analysis (right) showing the rising-edge shift between both materials; B. The $k^{3}$-wighted $\chi(r)$ Fe $K$ edge EXAFS spectra of Fe-0.5- and Fe-1.2-MOF-808 systems.

Density Functional Theory (DFT) calculations were performed in order to elucidate the possible conformations of the bimetallic core of the Fe-MOF-808. The structural and energetic properties of several binuclear iron-oxo and iron-hydroxo clusters deposited on the nodes of the MOF-808 were modelled. The approach in this study is similar to previous works, where the deposition of small copper-, cobalt- and nickel-(hydro)oxo clusters was investigated on the nodes of the NU-1000. ${ }^{24,28,29}$ An exhaustive description of the possible iron(hy)droxo clusters explored can be found in the ESI S10. Among the possible structures investigated, two possess structural features that agree with the experimental PDF. Moreover, from a thermodynamic point of view, they are the most stable conformations because of the most negative computed free energies of formation. In both of them, the bimetallic core is deposited as $-\mathrm{Fe}_{2} \mathrm{Cl}_{2}(\mu-\mathrm{OH})_{2}$ - on the nodes via the terminal-OH groups. In one case, the iron-hydroxo cluster is bridging two $\mathrm{Zr}_{6} \mathrm{O}_{8}$ octahedra (model A), while in the second case (model B) it is deposited on one node and the cluster is terminated by an additional hydroxo group (Fig. 4). The presence of an intense dPDF signal at $c a$. $3.3 \AA$, linked to $\mathrm{Fe} \cdots \mathrm{Fe}$ and $\mathrm{Fe} \cdots \mathrm{Zr}$ distances within model $A$, demonstrated the stabilization of the Fe-oxo clusters by bridging two $\mathrm{Zr}_{6} \mathrm{O}_{8}$ clusters as main species. Additionally, the occurrence of signals at $c a$. 3.0 and $c a$. $3.7 \AA$, associated with $\mathrm{Fe} \cdots \mathrm{Fe}$ and $\mathrm{Fe} \cdots \mathrm{Zr}$ distances within the model $\mathrm{B}$, evidenced the deposition of the Fe-oxo clusters within the $\mathrm{Zr}_{6} \mathrm{O}_{8}$ cluster trough terminal hydroxo groups. Remarkably, these results demonstrated that the Fe-oxo clusters are stabilized by bridging two neighbouring $\mathrm{Zr}_{6} \mathrm{O}_{8}$ nodes within MOF-808, as previously identified for other zirconia-based MOF systems. ${ }^{24}$

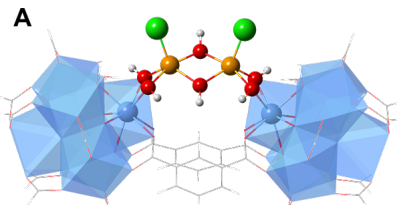

$\Delta \mathrm{G}_{\text {form }}=-221.9 \mathrm{~kJ} / \mathrm{mo}$

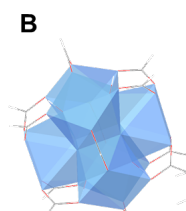

$\Delta \mathrm{G}_{\text {form }}=-165.7 \mathrm{~kJ} / \mathrm{mol}$
Fig. 4. DFT computed structures of possible bimetallic iron-hydroxo clusters deposited on the MOF-808. A. Iron cluster acts as bridge between two Zr-nodes. B. Iron cluster is linked to one Zr-node only with a terminal disposition. Their M06-L computed free energies of formation are also given. A more detailed description of the calculations can be found in the ESI.

As a proof of concept, activated Fe-MOF-808 materials were evaluated for the capture and subsequent catalytic degradation of BPA through Fenton reactions. The experiments were performed using solutions containing Milli-Q water and ethanol in a 95 to 5 ratio (see ESI, S11) at room temperature. Under these conditions, Fe-0.5-MOF-808 was able to capture ca. $50 \%$ of BPA after $1 \mathrm{~h}$, contrary to the $c a .11 \%$ captured by Fe-1.2MOF-808 (Fig. 5). This result is in accordance with the textural properties of the materials (Fig. 1A-B)- the MOF with a lower iron content (i.e. Fe-0.5-MOF-808) has larger free space to capture molecules than its analogue with higher iron content (i.e. Fe-1.2-MOF-808). To study the performance of the FeMOF-808 materials as catalysts, the degradation of BPA was carried out through a Fenton reaction using $\mathrm{H}_{2} \mathrm{O}_{2}$ (see details in ESI). The catalytic degradation was compared at 30 and $60 \mathrm{~min}$ reaction time (Fig. 5). Interestingly, the structural differences seen for the two MOFs play also a role in their catalytic performance regarding both the kinetic and the thermodynamic aspects. Thus, Fe-0.5-MOF-808 degraded the $26 \%$ of the total BPA after 30 minutes, being this value three times better than the activity determined for Fe-1.2-MOF-808. After 1 hour of reaction time, a plateau is reached and ca. $40 \%$ of the total BPA is removed by the low Fe-loaded material, being 
this value remarkably larger than the performance observed for Fe-1.2-MOF-808 containing larger iron-oxo clusters.

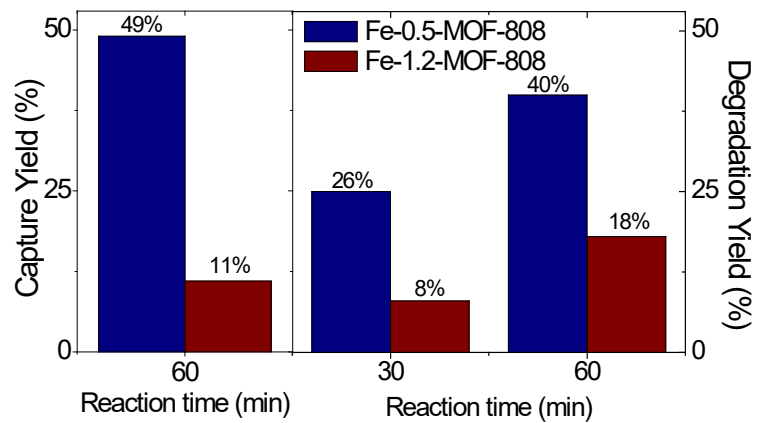

Fig. 5 Removal test of $1 \mathrm{mg} / 1 \mathrm{~mL} 100 \mathrm{ppm}$ BPA after $60 \mathrm{~min}$ (left), and catalytic degradation, $0.04 \%$ molar in Fe over 5 mL BPA 100 ppm (right).

In conclusion, we have reported the stabilization of catalytic Feoxo clusters within the MOF-808 through SIM methods, active in the degradation of BPA via Fenton reactions. Interestingly, synchrotron XAFS and PDF characterization combined with DFT modelling demonstrated the occurrence of Fe-oxo dimers bridging two neighbouring $\mathrm{Zr}_{6} \mathrm{O}_{8}$ nodes as the main species. We hypothesize that the terminal Fe-oxo species (model B) might be formed under kinetic control at short reaction times, and subsequent react with a terminal hydroxo group from a neighbouring zirconia node giving the formation of the most thermodynamically stable bridging species (model A). Further studies are currently under investigation to elucidate the structural mechanism of this Fe-oxo cluster stabilization.

This work was supported by the Spanish Government (RTI2018096138-A-I00 and RTI2018-101049-BI00) and the Regional Government of Madrid (TALENTO grants 2017-T1/IND5148 and 2017-T1/AMB-5264) for funding. We acknowledge the computing facilities of CSUC for providing computational resources. XAS experiments were performed at BL22 beamline (proposal 2019093796) at ALBA Synchrotron with the collaboration of ALBA staff. We thank DESY and Dr M. Wharmby for assistance in using the beamline P02.1 for the PDF experiments (proposal I-20190239 EC). The research leading to this result has been supported by the project CALIPSOplus

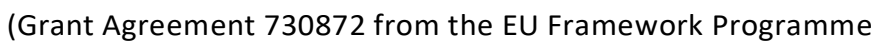
for Research and Innovation HORIZON 2020). C. C. B acknowledges the European Social Funds and the Regional Government of Madrid for a postdoctoral contract (PEJD-2018POST/IND-7909). I. R. M. acknowledges the Universidad Autónoma de Madrid for predoctoral fellowship.

\section{Conflicts of interest}

There are no conflicts to declare.

\section{Notes and references}

1 M. A. La Merrill, L. N. Vandenberg, M. T. Smith, W. Goodson, P. Browne, H. B. Patisaul, K. Z. Guyton, A. Kortenkamp, V. J. Cogliano, T. J. Woodruff, L. Rieswijk, H. Sone, K. S. Korach, A. C. Gore, L. Zeise and R. T. Zoeller, Nat. Rev. Endocrinol., 2020, 16, 45-57.

2 Q. Xie, J. Xie, Z. Wang, D. Wu, Z. Zhang and H. Kong, Microporous
Mesoporous Mater., 2013, 179, 144-150.

3 Y. Jeong, M. Cui, J. Choi, Y. Lee, J. Kim, Y. Son and J. Khim, Chemosphere, 2020, 238, 124559.

4 M. Munoz, P. Domínguez, Z. M. de Pedro, J. A. Casas and J. J. Rodriguez, Appl. Catal. B Environ., 2015, 203, 166-173.

5 Y. Li, T. Yang, S. Qiu, W. Lin, J. Yan, S. Fan and Q. Zhou, Chem. Eng. J., 2020, 389, 124382.

6 T. Devic and C. Serre, Chem. Soc. Rev., 2014, 43, 6097-6115.

7 E. Barea, C. Montoro and J. A. R. Navarro, Chem. Soc. Rev., 2014, 43, 54195430.

8 F. A. Son, M. C. Wasson, T. Islamoglu, Z. Chen, X. Gong, S. L. Hanna, J. Lyu, X. Wang, K. B. Idrees, J. J. Mahle, G. W. Peterson and O. K. Farha, Chem. Mater., 2020, 32, 4609-4617.

9 H. G. T. Ly, G. Fu, A. Kondinski, B. Bueken, D. De Vos and T. N. Parac-Vogt, J. Am. Chem. Soc., 2018, 140, 6325-6335.

10 Y. Bai, Y. Dou, L.-H. Xie, W. Rutledge, J.-R. Li and H.-C. Zhou, Chem. Soc. Rev., 2016, 45, 2327-2367.

11 R. Gil-San-Millan, E. López-Maya, A. E. Platero-Prats, V. Torres-Pérez, P. Delgado, A. W. Augustyniak, M. K. Kim, H. W. Lee, S. G. Ryu and J. A. R. Navarro, J. Am. Chem. Soc., 2019, 141, 11801-11805.

12 J. Baek, B. Rungtaweevoranit, X. Pei, M. Park, S. C. Fakra, Y. S. Liu, R. Matheu, S. A. Alshmimri, S. Alshehri, C. A. Trickett, G. A. Somorjai and O. M. Yaghi, J. Am. Chem. Soc., 2018, 140, 18208-18216.

13 J. Jiang, F. Gándara, Y.-B. Zhang, K. Na, O. M. Yaghi and W. G. Klemperer, J. Am. Chem. Soc., 2014, 136, 12844-12847.

14 N. Van Velthoven, S. Waitschat, S. M. Chavan, P. Liu, S. Smolders, J. Vercammen, B. Bueken, S. Bals, K. P. Lillerud, N. Stock and D. E. De Vos, Chem. Sci., 2019, 10, 3616-3622.

15 J. Liu, L. R. Redfern, Y. Liao, T. Islamoglu, A. Atilgan, O. K. Farha and J. T. Hupp, ACS Appl. Mater. Interfaces, 2019, 11, 47822-47829.

16 Z. Li, A. W. Peters, A. E. Platero-Prats, J. Liu, C. W. Kung, H. Noh, M. R. DeStefano, N. M. Schweitzer, K. W. Chapman, J. T. Hupp and O. K. Farha, J. Am. Chem. Soc., 2017, 139, 15251-15258.

17 M. Vandichel, J. Hajek, F. Vermoortele, M. Waroquier, D. E. De Vos and V. Van Speybroeck, CrystEngComm, 2015, 17, 395-406

18 A. E. Platero-Prats, A. Mavrandonakis, L. C. Gallington, Y. Liu, J. T. Hupp O. K. Farha, C. J. Cramer and K. W. Chapman, J. Am. Chem. Soc., 2016, 138, 4178-4185.

19 H. Xu, S. Sommer, N. L. N. Broge, J. Gao and B. B. Iversen, Chem. - A Eur. J., 2019, 25, 2051-2058.

20 C. Castillo-Blas, J. M. Moreno, I. Romero-Muñiz and A. E. Platero-Prats, Nanoscale, 2020, 12, 15577-15587.

21 B. Jeon, Q. Van Overmeere, A. C. T. Van Duin and S. Ramanathan, Phys. Chem. Chem. Phys., 2013, 15, 1821-1830.

22 K. M. $\varnothing$. Jensen, H. L. Andersen, C. Tyrsted, E. D. Bøjesen, A. C. Dippel, N. Lock, S. J. L. Billinge, B. B. Iversen and M. Christensen, ACS Nano, 2014, 8, 10704-10714.

23 H. Furukawa, F. Gándara, Y. B. Zhang, J. Jiang, W. L. Queen, M. R. Hudson and O. M. Yaghi, J. Am. Chem. Soc., 2014, 136, 4369-4381.

24 A. E. Platero-Prats, A. B. League, V. Bernales, J. Ye, L. C. Gallington, A. Vjunov, N. M. Schweitzer, Z. Li, J. Zheng, B. L. Mehdi, A. J. Stevens, A. Dohnalkova, M. Balasubramanian, O. K. Farha, J. T. Hupp, N. D. Browning, J. L. Fulton, D. M. Camaioni, J. A. Lercher, D. G. Truhlar, L. Gagliardi, C. J. Cramer and K. W. Chapman, J. Am. Chem. Soc., 2017, 139, 10410-10418.

25 J. K. Kowalska, A. W. Hahn, A. Albers, C. E. Schiewer, R. Bjornsson, F. A. Lima, F. Meyer and S. DeBeer, Inorg. Chem., 2016, 55, 4485-4497.

26 T. E. Westre, P. Kennepohl, J. G. DeWitt, B. Hedman, K. O. Hodgson and E. I. Solomon, J. Am. Chem. Soc., 1997, 119, 6297-6314.

27 R. N. Collins, K. M. Rosso, A. L. Rose, C. J. Glover and T. David Waite, Geochim. Cosmochim. Acta, 2016, 177, 150-169.

28 Z. Li, A. W. Peters, V. Bernales, M. A. Ortuño, N. M. Schweitzer, M. R. Destefano, L. C. Gallington, A. E. Platero-Prats, K. W. Chapman, C. J. Cramer, L. Gagliardi, J. T. Hupp and O. K. Farha, ACS Cent. Sci., 2017, 3, 31-38.

29 J. Zheng, J. Ye, M. A. Ortuño, J. L. Fulton, O. Y. Gutiérrez, D. M. Camaioni, R. K. Motkuri, Z. Li, T. E. Webber, B. L. Mehdi, N. D. Browning, R. L. Penn, O. K. Farha, J. T. Hupp, D. G. Truhlar, C. J. Cramer and J. A. Lercher, J. Am. Chem. Soc., 2019, 141, 9292-9304. 